\title{
Amary llidaceae Perspectives In Alzheimer'S Disease
}

\author{
Willian Orlando Castillo ${ }^{1}$, Andres Felipe Aristizabal-Pachon ${ }^{2}$, \\ Catarina Takahashi ${ }^{3}$ \\ ${ }^{1}$ (Department of Genetics, Ribeirao Preto Medical School, USP.wocastillo@usp.br) \\ ${ }^{2}$ (Department of Genetics, Ribeirao Preto Medical School, USP) \\ ${ }_{3}^{3}$ Department of Biology, Faculty of Philosophy, Sciences and Letters, Ribeirao Preto, USP)
}

\begin{abstract}
Alzheimer's disease (AD) is the most prominent type of dementia in elderly population. The etiology is multifactorial, and pathophysiology of disease is complex with slowly progressive and irreversible deterioration. Traditionally, AD researches have focused on the pathogenesis caused by Neuritic Plaques (NPs) and Neurofibrilary Tangles (NFTs); however, in the pathologic spectrum of disease, there are others independent pathways involved. Although several genetic alterations have been associated with AD, as memory as $\mathrm{AD}$ seem to be influenced by genetic, physiologic and environmental factors, resulted of accumulate over time. The current therapeutic approaches for AD temporarily improve the symptoms; and despite intensive efforts, none of the treatments available today alter the course of disease. Nevertheless, one of the most promising approaches for treating it is to enhance acetylcholine level and decrease oxidative stress in brain of AD patients. In line with this, different studies indicate that the alkaloids belonging to Amaryllidaceae family exhibit a wide range of biological activities. Galantamine has become the most attractive of alkaloids for its use in the treatment of $\mathrm{AD}$; however, Amaryllidaceaes contain other alkaloids which have high potential as acetylcholinesterase inhibitors (ACHEI) and antioxidant.
\end{abstract}

Keywords - ACHEI, Alzheimer's Disease, Amaryllidaceae, Galantamine.

\section{INTRODUCTION}

$\mathrm{AD}$ is a chronic progressive multifactorial neurodegenerative disorder, characterized by the irreversible deterioration of functions such as memory, language and other cognitive functions [1,2]. After the age of 65 years, the risk of developing the disease doubles every five years, and some studies suggest that around 85 years, approximately $50 \%$ of individuals will develop the disease [3]. Worldwide, one new case occurs every seven seconds; the disease itself is becoming a slow pandemic [4] and by the year 2050 it is expected that one in 85 persons will be living with AD [5]. Pathological changes in the brain of a patient with Alzheimer can start twenty years or more, before clinical symptoms are detectable [6] and the diagnosis is based on clinical and family history of the patient, beyond the cognitive performance in various psychometric tests. However, definitive diagnosis can be made only after death, through a brain autopsy.

Neuropathologically the brain of patients with $\mathrm{AD}$ is characterized by two classic markers that define the neuropathological features of the disease: the neuritic plaques (NPs) and neurofibrillary tangles (NTs) [7]. Pioneering investigations of NP and NT led to the identification of the molecules comprising these lesions, the amyloid beta peptide $(\mathrm{A} \beta)$ and tau proteins, respectively. NPs are extracellular aggregates containing $A \beta_{(1-42)}$ peptide as the major core deposit. $\mathrm{A} \beta_{(1-42)}$ is a peptide fragment derived through sequential cleavage from an integral membrane protein known as amyloid precursor protein (APP) by the action of various secretases [8]. An event in $\mathrm{AD}$ is the conversion of the soluble $\mathrm{A} \beta_{(1-42)}$ peptide to the aggregate form [9]. Initially, the original hypothesis attributed AD to the presence of insoluble A $\beta$ peptide in NPs [10], however, it has been more than 10 years since the hypothesis was modified with the suggestion that soluble oligomers of $A \beta$ peptide derived from the abnormal processing of APP are more toxic products than the same NPs [11].

In parallel, the intracellular deposits of NTs reflect abnormal modifications of the isoforms of microtubule-associated tau protein[12]. Natural cells commonly express tau protein, where its purpose is to stabilize the microtubules and this way, imparting shape and structure to the cell and generate a complex system of transport that allow movement and transmission of micronutrients, organelles and neurotransmitters [13]. However, tau phosphorylation is a key event in AD. Tau protein hyperphosphorylation in NTs lead to dissociation of tubulin, resulting in breakdown of microtubules and the degeneration in brain transport network [14]. One of the primary enzymes involved in tau phosphorylation has been focused on glycogen synthase kinase 3 (GSK3). Previous studies evaluating inhibitory action on GSK3 observed a correlation with reduced tauopathy and degeneration in a variety of animal models, in fact, GSK3 gene was previously associated with cancer [15-17]. In Alzheimer's conditions, the structural changes caused by tau hyperphosphorylation, interfere with the normal neurons function, lead to loss of biological activity and cell death [18]. Likewise, tau 
hyperphosphorylation may lead to defects in the mitotic spindle and result in a number of aneuploidies of chromosome 17 and 21, together with abnormal expression of tau protein, APP and A $\beta$ peptide, which are discussed in terms of its association with Alzheimer [19]. Considering all of this evidence, it seems that changes in tau weaken the links that stabilize microtubules and generate aggregation of the protein into the neurons. Therefore, deterioration in AD appears to be driven by both NPs and NFTs at different stages of the disease [20].

\section{NEUROPATHOLOGY OF AD}

$1.1 \mathrm{~A} \beta_{(1-42)}$ peptide a causal role in the pathogenesis of $\mathrm{AD}$.

Many hypotheses try to explain the extracellular deposits of $A \beta_{(1-42)}$ peptide which can be associated with onset of neurodegenerative cascade of events that result in synaptic dysfunction, cognitive impairment, neuronal loss and brain atrophy [21]. According to the amyloid hypothesis, AD begins with the abnormal processing of APP, which is cleaved by $\beta$ and $\gamma$ secretases through an amyloidogenic pathway. Additionally, this process originates a family of $A \beta$ peptides, including $A \beta_{(1-40)}$ and $A \beta_{(1-42)}$ peptides. $A \beta_{(1-42)}$ peptide is the most insoluble and the major component of the NPs which generates fibrillar aggregates, a pathological hallmark characteristic of disease [22]. Under physiological conditions, APP, a cell surface protein with a length of 695770 amino acids, is cleaved by $\alpha$ and $\gamma$ secretase and its originate a harmless peptide fragment of 40 amino acids known as P3 [23]. However, in the amyloidogenic pathway, the sequence $A \beta_{(1-42)}$ peptide is generated from APP by sequential cleavages by $\beta$ and $\gamma$ secretases (Fig. 1). $\beta$-secretase has been identified as BACE1 enzyme and $\gamma$ secretase is a multi-protein complex that involves at least four proteins including presenilin 1 (PSEN1), presenilin 2 (PSEN2), nicastrin (NCT) and Aph-1 which are required for a efficient proteolytic activity [22].

Although the causes of AD pathology largely remains unknown, several studies have been proposed to explain the relationship between the accumulation of the $A \beta_{(1-42)}$ peptide, change of microtubules by tau protein and oxidative stress [1]. Further, some studies suggest that metals such as $\mathrm{Cu}^{+2}$ and $\mathrm{Fe}^{+3}$ participate in the mechanism of oxidative stress induced by $A \beta_{(1-42)}$ peptide, mediated through of their redox potential. The transfer of one electron peptide to metal could result in the formation of a free radical peptide, which becomes a possible explanation for the formation of $A \beta$ radicals $[24,25]$. In addition, the interaction between $A \beta_{(1-42)}$ peptide and metal ions can generate reactive oxygen species (ROS) such as $\mathrm{H}_{2} \mathrm{O}_{2}$ which mediate cell neurotoxicity [26]. Oxidative stress and the neurotoxic mechanisms associated with the production of $A \beta$ radicals have been associated with the methionine residue in position 35 of $A \beta_{(1-42)}$ peptide [24]. It has been reported that wild type $A \beta_{(1-42)}$ peptide and its oxidized derivative carrying a methionine sulfoxide residue at position 35 showed the highest rate of fibril formation and exerted toxic activity. In contrast, truncated peptides, around the amino acid in position 35_showed a reduced aggregation rate, the immature fibers predominated, and the toxicity was of magnitude lower [27].

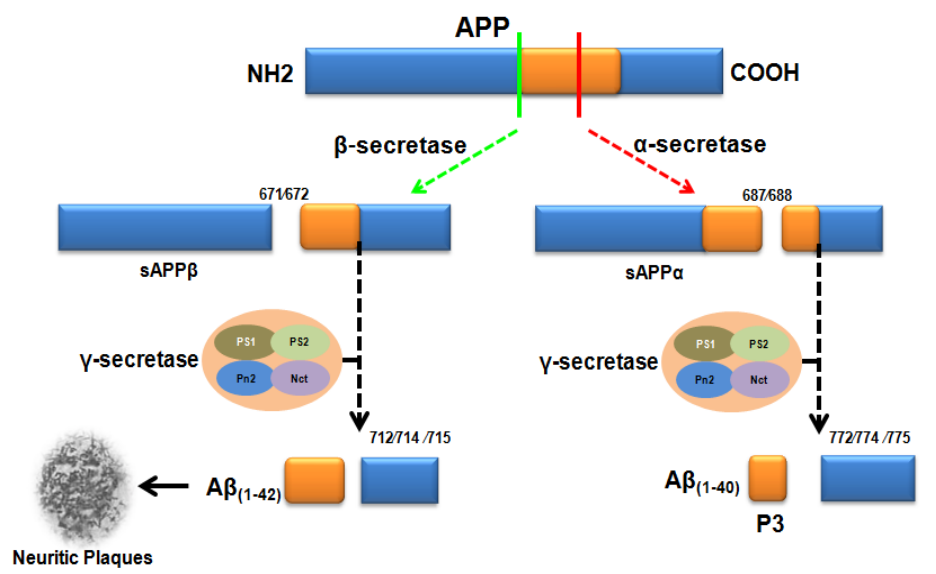

Figure 1. Non-amyloidogenic and amyloidogenic processing of APP. In physiological pathway, the APP is sequential cleavage by $\alpha$ and $\gamma$ secretase generating peptide fragments of 40 amino acids. However, through amyloidogenic pathway, sequential cleavage of APP by $\beta$ and $\gamma$ secretases originates different amyloid fragments, including the $\mathrm{A} \beta$ peptide fragment of 42 amino acids, which form the NPs.

According oxidative stress theory, neuronal death is a consequence of free radicals that bind and change the structural architecture of the lipid molecules of neurons. In this model, small soluble aggregates of the $\mathrm{A} \beta_{(1-42)}$ peptide could be inserted into the lipid bilayer of the cellular membrane of neurons and glial cells, then causing oxidative stress, including lipid peroxidation, proteins oxidation and DNA damage [25, 28]. 
Nevertheless, the pathologic changes include other biological alterations such as synapse loss, inflammation and neuronal death, resulting from vulnerability to stress and oxidative damage $[29,30]$. The exact mechanism by which the $A \beta_{(1-42)}$ peptide predominates and leads to neurotoxicity is still unknown [24]. Understand the pathways by which $A \beta_{(1-42)}$ peptide induces oxidative stress is essential in the association of the $A \beta$ peptide as a model of ROS for neurodegeneration in $\mathrm{AD}[25,28]$. Accordingly, the prevention of aggregation of $\mathrm{A} \beta$ plaques has been proposed as a primary therapeutic strategy for the treatment of neurodegenerative diseases such as Alzheimer [31]. Nevertheless, recent studies suggest that the oligomers of the $A \beta_{(1-42)}$ peptide are more toxic than their monomeric and fibrillary aggregates and although the molecular mechanisms that lead to the disease are not well understand, sufficient evidence regarding the role of oxidative stress and mitochondrial dysfunction in $\mathrm{AD}$ are recognized as early events in the development of the disease [32]. It has been shown that $\mathrm{A} \beta_{(1-42)}$ peptide gains access into mitochondrial matrix and its progressively accumulates mediates mitochondrial stress by interfering with enzyme activity, affecting the neural bioenergetics, an important pathway in the pathogenesis of this devastating disease $[33,34]$.

1.2 The cholinergic neurotransmission system in the brain

Another important feature in the brains of patients with AD is loss of cholinergic neurons and nicotinic acetylcholine receptors ( $\mathrm{AAChRs),} \mathrm{which} \mathrm{act} \mathrm{as} \mathrm{neuromodulators} \mathrm{in} \mathrm{cognitive} \mathrm{processes} \mathrm{regulated} \mathrm{by} \mathrm{various}$ neurotransmitters [35]. Dysfunction of the cholinergic system in AD patients taken place at different levels including a decrease in acetylcholine biosynthesis ( $\mathrm{ACh}$ ), transferase acetylcholine activity, reduced glucose uptake, reduction in choline metabolism and disrupting the function of acetylcholine receptors (AChRs) [36]. Different studies have shown that $A \beta_{(1-42)}$ peptide affects $n A C h R s$ function. These data suggest a significant reduction in the levels of nAChRs $\alpha 3, \alpha 7$ and $\beta 2$ protein subunits observed in cell lines exposed to peptide [37]. The progressive deterioration of the cholinergic system, together with the pharmacological evidence of AChEIs, have led to the development of cholinergic hypothesis, widely accepted and becomes evident by drugs such as donepezil, rivastigmine and galantamine, which act as AChEIs to increase the concentration of ACh between the synaptic cleft and modulate the nAChRs such as potent allosteric ligands [18].

1.3 Risk factors for AD

Neuropathological studies have shown that the most individuals diagnosed with sporadic AD do not have present the "pure" $\mathrm{AD}$ and the prevalence of comorbidity for diseases that contribute to cognitive impairment increases with advancing age [38]. AD may be classified based on the age of onset as early-onset $\mathrm{AD}$ and late-onset $\mathrm{AD}$. Early-onset $\mathrm{AD}$ accounts for approximately $1 \%$ to $6 \%$ of all cases and manifests roughly between 30 and 60 years, whereas that late-onset form accounting for around $90 \%$ of cases and it has an age at onset later than 60 years [13]. Although the greatest risk factor is advancing age, other factors are related, such as family history, cranial trauma, female gender, previous depression, diabetes mellitus, hyperlipidemia, vascular factors and oxidative stress [32]. However, it is known that many risk factors that contribute to the development of dementia in late life are modifiable [38]. On the other hand, mutations in three different genes are known to cause early-onset $\mathrm{AD}$ : amyloid beta $(\mathrm{A} \beta)$ precursor protein $(A P P)$, presenilin $1(P S E N 1)$ and presenilin 2 (PSEN2). The majority of these mutations appear to be dominantly inherited; however, not all are completely penetrant to the illness. Clinical features and pathology vary depending on the mutation's locus and position within each gene [39]. Many people may have the same risk factors for AD, expressing mutated A $\beta$ peptide, besides alterations in tau protein and never develop the disease [40]; which suggests, that these mechanisms together with the genetic association cannot fully explain the susceptibility to develop of the disease [41]. Additional genetic, epigenetic and environmental factors need to be identified in order to understand truly the development of these neurodegenerative disorders [42]. The dynamic nature of epigenetic marks and their involvement in adaptive processes different such as cell differentiation, age [43] and response to external stimuli make them very interesting epigenetic candidates to be involved in the etiology of complex diseases such as Alzheimer's. Experiences and environmental factors can critically influence the risk and progression of neurodegenerative disorders [42].

\section{AD TREATMENT: AMARYLLIDACEAE ALKALOIDS}

$\mathrm{AD}$ is known for about a century and despite intensive efforts of science, there is not an effective treatment that can modify or change the course of the pathology. However, statistics show an exponential rise in the number of cases with the disease. These findings emphasize the enormous and urgent need for develop an effective treatments [44]. Due to the complexity of the disease and increasing of population with advanced age, $\mathrm{AD}$ is the public health crisis of the $21^{\text {st }}$ century [45] and the current pharmacological therapies provide symptomatic improvement alone. Currently the drugs approved for the treatment of AD are classified into two groups: ACHEIs and N-Methyl-D-aspartate (NMDA) receptors [46]. There are four ACHEIs approved by FDA; tacrine, donepezil, rivastigmine and galantamine. However, tacrine is rarely used for its hepatotoxicity [47]. Some ACHEIs are derived from natural sources, e. g., galantamine, a natural alkaloid, isolated from bulbs and aerial parts of plants from the Amaryllidacea family [48]. 
Plants of the Amaryllidaceae family, a small group of monocotyledonous species, belong to the 20 most important alkaloid-containing plant families, which exhibit a wide range of interesting biological activities and some of them have been approved for clinical therapy, of which ACHEI activity is the most relevant [49]. This fact has motivated the screening of Amaryllidaceae alkaloids as possible ACHEI and the results have shown that galantamine and lycorine are superior such as ACHEIs with respect to other alkaloids [49, 50]. Galantamine commercialized under the generic name of Reminil® was the first alkaloid isolated from different species of Amaryllidaceae (Leucojum spp., Narcissus species, Galanthus spp) and is the most recently ACHEI approved in many countries for the symptomatic treatment of Alzheimer's disease [51]. However, the pharmacological history of galantamine shows that it has been used throughout Eastern Europe since 1950, and the plant extracts with this kind of active compound were initially used to treat nerve pain and poliomyelitis [52].Galantamine is a cholinergic drug with antioxidant properties; neuroprotective and anti-apoptotic action $[18,53]$ by its dual mechanism of action; moreover, it regulates the cholinergic transmission as ACHEI and allosterically modulates nAChRs [54]. The drug stimulates choline acetyltransferase activity and enhances the release of acetylcholine neurotransmitter, increasing its concentration in the synaptic clefts [53]. Galantamine reduces acetylcholinesterase (ACHE) levels, and increases nicotinic receptors binding, ameliorates learning deficits significantly during acquisition and retention [55].

The Cognitive impairment observed in patients with $\mathrm{AD}$ is reduced with galantamine treatment; however, does not always correlate with its action as ACHEI. Additionally to modulating nicotinic receptors, galantamine also modulates the muscarinic cholinergic system and reduces the levels of A $\beta_{(1-40)}$ and $A \beta$ (1-42) peptides in cell cultures and cerebrospinal fluid to directly interact with the peptide; in this way, diseasemodifying effects of the drug could be due to an additional mechanism on A $\beta$ peptide aggregation and/or toxicity [56]. Recent results have been shown that chronic treatment with galantamine was capable of delayed A $\beta$ plaque deposits and reduced gliosis in 5XFAD mouse. These results strongly support that galantamine, in addition to improving cognitive and behavioral symptoms in $\mathrm{AD}$, may have disease-modifying and neuroprotective properties [57].

The efficacy of galantamine has been shown for treatment of $\mathrm{AD}$ in mild, moderate and advanced moderate stages, however, recently has been reported its efficacy in patients with AD in severe stage [58]. Likewise, Galantamine also promotes hippocampal neurogenesis but the exact mechanism for this is not known nevertheless, it is believed that could be influenced by via activation of the M1 muscarinic and $\alpha 7$ nicotinic acetylcholine receptors and IGF2, stimulating the survival of immature cells in the granule cell layer [59]. In line with this, it has shown that IGF2 regulate proliferation of hypocampal neural stem cell through AKTdependent signal. AKT pathway appears to be involved in promotes neuronal survival, neuronal protection, stimulates anti-apoptotic signaling and inhibition autophagy, disruptions which play an import role in neurodegenerative disorders [60]. Some authors have proposed that neurogenesis may provide a natural defense strategy against the neurodegeneration as a novel therapeutic strategic for $\mathrm{AD}$, increasing neuroregeneration in the brain which may be achieved by stimulating the brain's endogenous stem cells in the major neurogenic zone, the subventricular zone (SVZ) in the lateral ventricles or in the dentate gyrus (DG) [7]. Cognitive impairment in $\mathrm{AD}$ patients has been associated with a decline in the levels of growth factors, impairment of axonal transport and marked degeneration of basal forebrain cholinergic neurons (BFCNs) [61].

On the other hand, due to the impact generated by disease and by the multiple pathways altered in AD patients, is a challenge for modern medicine finds novel therapeutic targets. Plants due to their different properties have the potential to prevent, delay or ameliorate many human disorders. In western medicine most of the drugs used for the treatment of neurodegenerative disorders are derived from plant sources [48]. The World Health Organization (WHO) has estimated that more than $75 \%$ of the world population depends on traditional medicine, based on the use of plants [62]. Besides, it has been published that total extracts have a better bioprotector system due to the synergistic interaction of its active compounds [63].

In order to contribute to a better understanding of the role of total plant extracts in Alzheimer's treatment, our laboratory has combined these concepts into a comprehensive cell model for neurotoxicity associated with the $\mathrm{A} \beta_{(1-42)}$ peptide and the possible neuroprotective effect of total extract of Caliphruria subedentata. Caliphruria is a genus of bulbou plants belongs to the Amaryllidaceae family that consists of four species: C. korsakofii (Traub), C. hartwegiana (Herb), C. subedentata (Baker) and C. tenera (Baker), which are distributed in tropical regions of South America. C. subedentata is endemic of Colombia and is considered an endangered specie [64]. In previews phytochemical studies, several alkaloids of this plant have been isolated and identified, among them, galantamina and lycorine which have high activity as AChEI and antioxidant [64].

Preliminary results in our laboratory (paper in preparation) have shown that the presence of $A \beta_{(1-42)}$ peptide in SH-SY5Y cell line lead to an significant increase of the cytotoxicity, DNA damage and decreased cell proliferation. Nevertheless, post-treatments with total extract of $C$ subedentata resulted in high neuroprotective activity against $A \beta_{(1-42)}$ peptide. Taken together, these findings may contribute to understand additional mechanisms, resulted of the synergistic interaction of constituents present in the total extracts of 
Amaryllidaceaes. The growing demand for galantamine has prompted searches for new sources of this compound, as well as other bioactive alkaloids for the treatment of AD. The multiple mechanisms involved in the pathogenesis of AD create considerable difficulty in producing an effective treatment [13]. Nevertheless, the plants may provide a window of opportunities as therapeutic strategies and the Amaryllidaceae alkaloids might act in the modulation of any of these unregulated pathways in AD pathology.

\section{CONCLUSION}

In conclusion, due to the great challenge facing the multiple pathways deregulated in AD; find an appropriate balance between the synergism exerted by plant metabolites (e.g. alkaloids), could provide better therapeutic profiles and identify new candidates for next generation drugs.

\section{REFERENCES}

[1] Mattson, M.P., Pathways towards and away from Alzheimer's disease. Nature. 430(7000) 2004, p. 631639.

[2] Kumar, A. and A. Singh, A review on Alzheimer's disease pathophysiology and its management: an update. Pharmacological Reports. 67(2) 2015, p. 195-203.

[3] Felsenstein, K.M., et al., Regenerative medicine in Alzheimer's disease. Translational Research. 163(4) 2014, p. 432-438.

[4] Ferri, C.P., et al., Global prevalence of dementia: a Delphi consensus study. The Lancet. 366(9503) 2006, p. 2112-2117.

[5] Brookmeyer, R., et al., Forecasting the global burden of Alzheimer's disease. Alzheimer's \& Dementia. 3(3) 2007, p. 186-191.

[6] Reiman, E.M., et al., Brain imaging and fluid biomarker analysis in young adults at genetic risk for autosomal dominant Alzheimer's disease in the presenilin 1 E280A kindred: a case-control study. The Lancet Neurology. 11(12) 2012, p. 1048-1056.

[7] Lilja, A.M., et al., Neurotrophic and Neuroprotective Actions of (-)-and (+)-Phenserine, Candidate Drugs for Alzheimer's Disease. PLoS One. 8(1) 2013, p. e54887.

[8] Ashe, K.H. and K.R. Zahs, Probing the biology of Alzheimer's disease in mice. Neuron. 66(5) 2010, p. 631-645.

[9] Selkoe, D.J., Alzheimer's Disease--Genotypes, Phenotype, and Treatments. Science. 275(5300) 1997, p. 630-631.

[10] Joachim, C.L. and D.J. Selkoe, The Seminal Role of [beta]-Amyloid in the Pathogenesis of Alzheimer Disease. Alzheimer Disease \& Associated Disorders. 6(1) 1992, p. 7-34.

[11] Rosenblum, W.I., Why Alzheimer trials fail: removing soluble oligomeric beta amyloid is essential, inconsistent, and difficult. Neurobiology of aging. 35(5) 2014, p. 969-974.

[12] Goedert, M., S.S. Sisodia, and D.L. Price, Neurofibrillary tangles and $\beta$-amyloid deposits in Alzheimer's disease. Current opinion in neurobiology. 1(3) 1991, p. 441-447.

[13] Anand, R., K.D. Gill, and A.A. Mahdi, Therapeutics of Alzheimer's disease: past, present and future. Neuropharmacology. 76 2014, p. 27-50.

[14] Iqbal, K., et al., Mechanisms of neurofibrillary degeneration and the formation of neurofibrillary tangles. J Neural Transm Suppl. 53 1998, p. 169-80.

[15] Domínguez, J.M., et al., Evidence for irreversible inhibition of glycogen synthase kinase-3 $\beta$ by tideglusib. Journal of Biological Chemistry. 287(2) 2012, p. 893-904.

[16] Aristizabal-Pachon, A.F., Complexo de Degradação de b-Catenina e o Câncer de mama (Novas Edições Acadêmicas: Saarbrüicke, 2015).

[17] Aristizabal-Pachon, A.F., et al., AXIN2 Polymorphisms, the beta-Catenin Destruction Complex Expression Profile and Breast Cancer Susceptibility. Asian Pac J Cancer Prev. 16(16) 2015, p. 7277-84.

[18] Arias, E., et al., Unequal neuroprotection afforded by the acetylcholinesterase inhibitors galantamine, donepezil, and rivastigmine in SH-SY5Y neuroblastoma cells: role of nicotinic receptors. Journal of Pharmacology and Experimental Therapeutics. 315(3) 2005, p. 1346-1353.

[19] Thomas, P. and M. Fenech, A review of genome mutation and Alzheimer's disease. Mutagenesis. 22(1) 2006, p. 15-33.

[20] Tiraboschi, P., et al., The importance of neuritic plaques and tangles to the development and evolution of AD. Neurology. 62(11) 2004, p. 1984-1989.

[21] Samanta, M.K., et al., Alzheimer disease and its management: a review. American journal of therapeutics. 13(6) 2006, p. 516-526.

[22] Giliberto, L., et al., Mutant presenilin 1 increases the expression and activity of BACE1. Journal of Biological Chemistry. 284(14) 2009, p. 9027-9038. 
[23] Selkoe, D.J., Alzheimer's disease: genes, proteins, and therapy. Physiological reviews. 81(2) 2001, p. 741-766.

[24] Varadarajan, S., et al., Different mechanisms of oxidative stress and neurotoxicity for Alzheimer's A $\beta$ (142) and A $\beta$ (25-35). Journal of the American Chemical Society. 123(24) 2001, p. 5625-5631.

[25] Varadarajan, S., et al., Review: Alzheimer's amyloid ß-peptide-associated free radical oxidative stress and neurotoxicity. J Struct Biol. 130(2-3) 2000, p. 184-208.

[26] Smith, D.G., R. Cappai, and K.J. Barnham, The redox chemistry of the Alzheimer's disease amyloid $\beta$ peptide. Biochimica et Biophysica Acta (BBA)-Biomembranes. 1768(8) 2007, p. 1976-1990.

[27] Seilheimer, B., et al., The toxicity of the Alzheimer's $\beta$-amyloid peptide correlates with a distinct fiber morphology. Journal of structural biology. 119(1) 1997, p. 59-71.

[28] Butterfield, D.A. and C.M. Lauderback, Lipid peroxidation and protein oxidation in Alzheimer's disease brain: potential causes and consequences involving amyloid $\beta$-peptide-associated free radical oxidative stress. Free Radical Biology and Medicine. 32(11) 2002, p. 1050-1060.

[29] Petrozzi, L., et al., Cytogenetic alterations in lymphocytes of Alzheimer's disease and Parkinson's disease patients. Neurological Sciences. 23(2) 2002, p. s97-s98.

[30] Thomas, P., et al., Grape seed polyphenols and curcumin reduce genomic instability events in a transgenic mouse model for Alzheimer's disease. Mutation Research/Fundamental and Molecular Mechanisms of Mutagenesis. 661(1-2) 2009, p. 25-34.

[31] Liu, R., et al., Trehalose differentially inhibits aggregation and neurotoxicity of beta-amyloid 40 and 42 . Neurobiology of disease. 20(1) 2005, p. 74-81.

[32] Kaur, N., et al., Oxidative Stress Events and Neuronal Dysfunction in Alzheimer's Disease: Focus on APE1/Ref-1-Mediated Survival Strategie (Springer. 2015).

[33] Chen, X. and S.D. Yan, Mitochondrial A $\beta$ A potential cause of metabolic dysfunction in Alzheimer's disease. IUBMB life. 58(12) 2006, p. 686-694.

[34] Mossmann, D., et al., Amyloid- $\beta$ Peptide Induces Mitochondrial Dysfunction by Inhibition of Preprotein Maturation. Cell metabolism. 20(4) 2014, p. 662-669.

[35] Nordberg, A., Nicotinic receptor abnormalities of Alzheimer's disease: therapeutic implications. Biological psychiatry. 49(3) 2001, p. 200-210.

[36] $\mathrm{Xu}, \mathrm{Y}$., et al., Neurotransmitter receptors and cognitive dysfunction in Alzheimer's disease and Parkinson's disease. Progress in neurobiology. 97(1) 2012, p. 1-13.

[37] Qi, X.-L., et al., Oxidative stress induced by beta-amyloid peptide is involved in the altered composition of cellular membrane lipids and the decreased expression of nicotinic receptors in human SH-SY5Y neuroblastoma cells. Neurochemistry international. 46(8) 2005, p. 613-621.

[38] Carrillo, M.C., et al., Can we prevent Alzheimer's disease? Secondary "prevention" trials in Alzheimer's disease. Alzheimer's \& Dementia. 9(2) 2013, p. 123-131. e1.

[39] Ridge, P.G., M.T. Ebbert, and J.S. Kauwe, Genetics of Alzheimer's disease. BioMed research international. 2013, p. 221-6.

[40] Lue, L.F., et al., Inflammation, A beta deposition, and neurofibrillary tangle formation as correlates of Alzheimer's disease neurodegeneration. Journal of neuropathology and experimental neurology. 55(10) 1996, p. 1083.

[41] Hamza, T.H. and H. Payami, The heritability of risk and age at onset of Parkinson's disease after accounting for known genetic risk factors. Journal of human genetics. 55(4) 2010, p. 241-243.

[42] Iraola-Guzmán, S., X. Estivill, and R. Rabionet, DNA methylation in neurodegenerative disorders: a missing link between genome and environment? Clinical genetics. 80(1) 2011, p. 1-14.

[43] Hernandez, D.G., et al., Distinct DNA methylation changes highly correlated with chronological age in the human brain. Human molecular genetics. 20(6) 2011, p. 1164-1172.

[44] Henriksen, K., et al., The future of blood-based biomarkers for Alzheimer's disease. Alzheimer's \& Dementia. 10(1) 2014, p. 115-131.

[45] Snyder, H.M., et al., Developing novel blood-based biomarkers for Alzheimer's disease. Alzheimer's \& Dementia. 10(1) 2014, p. 109-114.

[46] Racchi, M., et al., Acetylcholinesterase inhibitors: novel activities of old molecules. Pharmacological Research. 50(4) 2004, p. 441-451.

[47] Watkins, P.B., et al., Hepatotoxic effects of tacrine administration in patients with Alzheimer's disease. Jama. 271(13) 1994, p. 992-998.

[48] Tsvetkova, D., et al., Antioxidant activity of galantamine and some of its derivatives. Current medicinal chemistry. 20(36) 2013, p. 4595-4608.

[49] Cortes, N., et al., Neuroprotective activity and acetylcholinesterase inhibition of five Amaryllidaceae species: A comparative study. Life Sciences. 122 2015, p. 42-50. 
[50] Elgorashi, E.E., G.I. Stafford, and J. Van Staden, Acetylcholinesterase enzyme inhibitory effects of amaryllidaceae alkaloids. Planta medica. 70(3) 2004, p. 260-262.

[51] Heinrich, M. and H. Lee Teoh, Galanthamine from snowdrop-the development of a modern drug against Alzheimer's disease from local Caucasian knowledge. Journal of ethnopharmacology. 92(2) 2004, p. 147-162.

[52] Marco, L. and M.d.C. Carreiras, Galanthamine, a natural product for the treatment of Alzheimer's disease. Recent patents on CNS drug discovery. 1(1) 2006, p. 105-111.

[53] Arias, E., et al., Galantamine prevents apoptosis induced by $\beta$-amyloid and thapsigargin: involvement of nicotinic acetylcholine receptors. Neuropharmacology. 46(1) 2004, p. 103-114.

[54] Wang, D., et al., The allosteric potentiation of nicotinic acetylcholine receptors by galantamine ameliorates the cognitive dysfunction in beta amyloid25-35 icv-injected mice: involvement of dopaminergic systems. Neuropsychopharmacology. 32(6) 2007, p. 1261-1271.

[55] Woodruff-Pak, D.S., R.W. Vogel, and G.L. Wenk, Galantamine: effect on nicotinic receptor binding, acetylcholinesterase inhibition, and learning. Proceedings of the National Academy of Sciences. 98(4) 2001, p. 2089-2094.

[56] Raskind, M.A., et al., The cognitive benefits of galantamine are sustained for at least 36 months: a longterm extension trial. Arch Neurol. 61(2) 2004, p. 252-256.

[57] Bhattacharya, S., et al., Galantamine Slows Down Plaque Formation and Behavioral Decline in the 5XFAD Mouse Model of Alzheimer's Disease. PLoS One. 9(2) 2014, p. e89454.

[58] Burns, A., et al., Safety and efficacy of galantamine (Reminyl) in severe Alzheimer's disease (the SERAD study): a randomised, placebo-controlled, double-blind trial. Lancet Neurol. 8(1) 2009, p. 39-47.

[59] Kita, Y., et al., Galantamine promotes adult hippocampal neurogenesis via M1 muscarinic and $\alpha 7$ nicotinic receptors in mice. International Journal of Neuropsychopharmacology. 17(12) 2014, p. 19571968.

[60] Heras-Sandoval, D., et al., The role of PI3K/AKT/mTOR pathway in the modulation of autophagy and the clearance of protein aggregates in neurodegeneration. Cellular signalling. 26(12) 2014, p. 2694-2701.

[61] Wicklund, L., et al., B-amyloid 1-42 oligomers impair function of human embryonic stem cell-derived forebrain cholinergic neurons. PLoS One. 5(12) 2010, p. e15600.

[62] Organization, W.H., WHO traditional medicine strategy 2002-2005. 2002, p.

[63] Wu, T., et al., In vitro studies of Gynura divaricata (L.) DC extracts as inhibitors of key enzymes relevant for type 2 diabetes and hypertension. Journal of ethnopharmacology, 2011, p. 112-6.

[64] Cabezas, F., et al., Análisis del contenido alcaloidico de Caliphruria Subdentata Baker (Amaryllidaceae) por el método CG-EM. Revista latinoamericana de química. 41(1) 2013, p. 68-73. 